BI-TP 2003/28

BNL-NT-03/29

\title{
Free Meson Spectral Functions on the Lattice*
}

\author{
S. Stickan, ${ }^{a}$ F. Karsch, ${ }^{a}$ E. Laermann, ${ }^{a}$ P. Petreczky ${ }^{b}$ \\ ${ }^{a}$ Fakultät für Physik, Universität Bielefeld, D-33615 Bielefeld, Germany \\ bNuclear Theory Group, Physics Department Brookhaven National Laboratory, Upton NY 11973
}

We present results from an analytic calculation of thermal meson spectral functions in the infinite temperature (free field) limit. We compare spectral functions for various lattice fermion formulations used at present in studies of in-medium properties of hadrons based on the maximum entropy method (MEM). In particular, we will present a new calculation of spectral functions performed with extended quark sources.

\section{Introduction}

The modification of in-medium properties of mesons is one of the crucial concepts explored in experimental studies of hot and dense matter in created in heavy ion collisions. It is the hope that the creation of a dense matter and eventually also the quark gluon plasma can be unambiguously demonstrated through the study of modifications generated in the spectrum of heavy $(J / \psi$ suppression) as well as light quark bound states (shift of the $\rho$-meson mass, resonance broadening, thermal dilepton enhancement,..).

Lattice calculations can contribute to the theoretical discussion of these burning experimental questions through the calculation of thermal spectral functions, which control the structure of hadron correlation functions at finite temperature. The reconstruction of spectral functions from hadron correlation functions calculated on the lattice at finite temperature is based on a statistical tool, the Maximum Entropy Method (MEM) [1-4] which has been explored and tested over recent years. We will discuss here results of an analytic calculation, which becomes feasible in the infinite temperature limit which corresponds to a free fermion theory. In addition to calculations on isotropic and anisotropic lattices based on hadronic currents constructed from point sources we will present a new calculation of spectral functions performed for extended quark

\footnotetext{
*This work is supported by BMBF under grant No.06BI102 and DFG under grant FOR 339/2-1. P.P. is Goldhaber fellow supported by the contract DE-AC0298CH10886 with the U.S. Department of Energy.
}

sources.

\section{Free Lattice Spectral Functions}

The calculation of hadronic spectral functions (SPF) on the lattice starts from the Euclidian correlation function of hadronic currents, $G_{H}(\tau)$, with given quantum numbers, $H$. In the infinite temperature limit these correlation functions (CF) will approach the free fermion $\mathrm{CF}$, which can be expressed in terms of the free lattice quark propagator $S_{L}$. We will discuss here analytic calculations for spectral functions determined from correlation functions defined with currents constructed from point-like (p) as well as extended (exponentially smeared $e \equiv \exp \left(-x^{2}\right)$ or fuzzed $R=1$ ) sources $[5,6]$. Furthermore, we will present results on isotropic as well as anisotropic lattices. The anisotropy in the spatial $(a)$ and temporal $\left(a_{\tau}\right)$ lattice spacings is controlled by the anisotropy parameter $\xi \equiv a / a_{\tau}$. While the calculations on anisotropic lattices have been performed for the case of a Wilson action with $r$ parameter being set to unity as well as for the case of Wilson fermions where the time-like $r$ parameter is linked to the anisotropy $r=1 / \xi$. This latter choice is often used in heavy quark simulations and has also been used in the MEM analysis of heavy quark bound states [5]. In addition we present results from calculations with a truncated fixed-point action [7]. In momentum space the Euclidean time correlation functions for 
free fermions is then given by,

$$
\begin{aligned}
G_{H}^{L}(\tau) & =\int w_{s}(\mathbf{k}) \operatorname{Tr}\left[\Gamma_{H} S_{L}(\tau, \mathbf{k}) \Gamma_{H}^{\dagger} S_{L}^{\dagger}(\tau, \mathbf{k})\right] \mathrm{d}^{3} k \\
& =\int \sigma_{H}^{L}(\omega, T) \frac{\operatorname{ch}(\omega(\tau-1 / 2 T))}{\operatorname{sh}(\omega / 2 T)} \mathrm{d} \omega, \\
w_{s}(\mathbf{k}) & =\left\{\begin{array}{l}
1 \\
\exp \left(\mathbf{k}^{2} / 4\right) \\
\exp \left(\mathbf{k}^{2} / 2\right) \\
\cos (\mathbf{k R})
\end{array} \text { for } s=\left\{\begin{array}{l}
p p \\
e p \\
e e \\
R=1
\end{array},\right.\right.
\end{aligned}
$$

where $\Gamma_{H}$ is a product of $\gamma$-matrices that fixes the quantum numbers $H$. The crucial step in Eq.(1) is the second equality where the 3-dimensional momentum integral has been replaced by a 1dimensional integral over the energy $\omega$. Two further integrations are hidden in the definition of the spectral function $\sigma_{H}^{L}(\omega, T)$. We note that a separation of the dependence on Euclidean time $\tau$ in the integration kernel,

$K(\omega, \tau)=\operatorname{ch}(\omega(\tau-1 / 2 T)) / \operatorname{sh}(\omega / 2 T)$,

which kept its continuum form, and the lattice spectral function $\sigma_{H}^{L}(\omega, T)$, which is $\tau$ independent and carries the entire cut-off dependence of the CFs, could be achieved. This makes the introduction of a lattice kernel, which has been used in some numerical studies based on the MEM analysis [1,2] questionable. The analytic calculations are presented in [8] for pointlike sources and the truncated fixed-point action. The resulting spectral functions obtained in these cases on lattices with different anisotropies are shown in Fig. 1.

A closer look at the resulting SPFs shows that they all agree quite well with the continuum result,

$\sigma_{\text {cont }}(\omega, T)=\frac{3}{8 \pi^{2}} \omega^{2} \tanh (\omega / T)$

in the IR regime. This agreement extends to larger energies for the Wilson action on anisotropic lattices and even more so for the truncated fixed-point action [7]. The choice $r=1 / \xi$ apparently leads to a very abrupt deviation from this continuum like behavior and leads to large

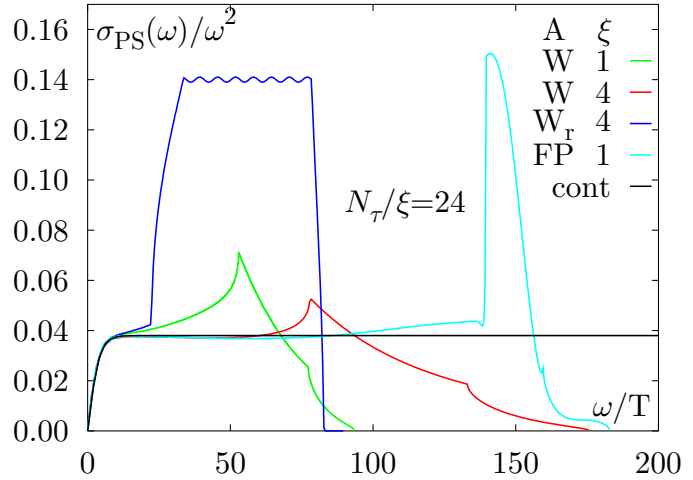

Figure 1. The free pseudo-scalar SPF for the Wilson action $(\mathrm{W})$ on isotropic $(\xi=1)$ and anisotropic $(\xi=4)$ lattices, the Wilson action with the choice $r=1 / \xi=4\left(W_{r}\right)$ and a truncated fixed-point action (FP). A part of the SPF for $W_{r}$ has been cut out at intermediate energies for better visibility.

cut-off effects for $\omega / T \geq 10$. This makes its use for the heavy quark spectroscopy at high temperature questionable. The other actions also show the influence of lattice artefacts in the UV region. Lattice artefacts show up as peaks and cusps which can directly be related to the sudden restriction of the available momentum phase space near the corners of the Brillouin zone for the quark dispersion relation. A close relation between the quark dispersion relation and the structure of SPFs thus is obvious in the free theory.

\section{Extended Sources}

To enhance the overlap with the ground state excitation in a given quantum number channel, extended operators are often used. Above $T_{c}$, the existence of a well separated ground state is, however, not guaranteed and it thus is questionable whether extended operators can be of any use at all. Nevertheless, extended operators have been used in the MEM analysis of meson correlation functions [5]. As they reduce to point like operators in the continuum limit, when their extend is kept fixed in lattice units, they may indeed also be suitable for studies of spectral functions. We 


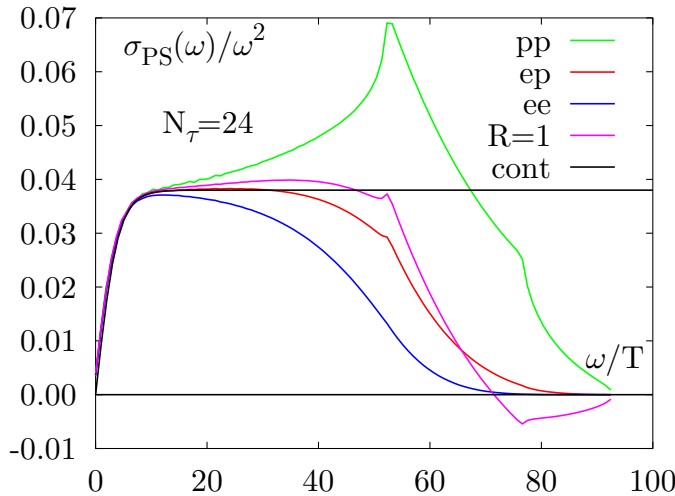

Figure 2. The pseudo-scalar SPF calculated on isotropic lattices using for different extended meson operators as defined in Eq.(2).

thus have analyzed some of them also in the free field limit. The SPFs of extended operators can be easily obtained using the so called "binning procedure" (see [8] for details). Some results are shown in Fig. 2. As can be seen the UV regime indeed is strongly suppressed and a good agreement with the continuum spectral function can be achieved of a wide range of energies up to the region where the point-like currents lead to large contributions from the "Wilson doublers". This suppression is more effective for operators with larger extend, as can be observed with the (ee) smeared operator which in the considered limit can also be viewed as a broader smeared exponential $(e p)$ operator. We note, however, that the use of extended sources requires tuning of parameters and care has to be taken that the low energy part does not get to be suppressed too, which happen easily with the $(e e)$ operator. Some versions of extended operators also violate the positivity of the spectral functions, which enters as a basic assumption in the MEM analysis [1]. The fuzzed $(R=1)$-source has this problem for $\omega / T \geq 70$. Moreover, the exponentially smeared sources are not gauge invariant and gauge fixing becomes necessary. For the application in simulations this additional computational overhead has to be compared with the improvement which could be achieved by using improved fermion ac- tions or by increasing $N_{\tau}$.

\section{Conclusions}

The analysis of the free lattice SPF provides basic information on the structure of spectral functions reconstructed in the interacting case from a statistical analysis based on MEM. It also can provide guidance for a suitable choice of the default model in the MEM analysis and the choice of an appropriate integration kernel in the spectral representation. In the free field limit lattice artefacts only lead to a modification of the SPF, the integration kernel keeps its continuum form. Extended meson operators lead to an efficient suppression of the UV part of the SPFs. This, however, requires a careful choice of parameters in order not to influence also the IR part of the spectral functions which at finite temperature is not well separated from the short distance part of Euclidean correlation functions.

\section{REFERENCES}

1. M. Asakawa et al., Prog. Part. Nucl. Phys. 46, (2001) 459

2. F. Karsch et al., Phys. Lett. B 530 (2002) 147

3. M. Asakawa et al., Nucl. Phys. A 715, (2003) 863

4. M. Asakawa and T. Hatsuda, hep-lat/0309001

5. T. Umeda et al., hep-lat/0211003

6. P. Lacock et al., Phys. Rev. D 51,(1995) 6403

7. W. Bietenholz et al., Nucl. Phys. Proc. Suppl. 106, (2002) 510

8. F. Karsch et al., Phys. Rev. D 68, (2003) 014504 